\title{
Emerging treatment options for patients with recurrent advanced thymic epithelial tumors
}

This article was published in the following Dove Press journal:

OncoTargets and Therapy

6 September 2012

Number of times this article has been viewed

\section{Tracey L Evans}

Abramson Cancer Center, University of Pennsylvania, Philadelphia, PA, USA
Correspondence: Tracey Evans Abramson Cancer Center, University of Pennsylvania,

2 Perelman, 3400 Civic Center Blvd,

Philadelphia, PA 19104, USA

$\mathrm{Tel}+\mathrm{I} 215662668 \mathrm{I}$

Fax +I 2156155888

Email tracey.evans@uphs.upenn.edu
Abstract: The purpose of this review article is to review recent advances in the treatment of advanced thymic epithelial tumors. These tumors are generally responsive to cytotoxic combination chemotherapy in the first-line setting. While newer agents have shown efficacy in the salvage setting, there is no one standard approach. A multitude of targeted agents have shown promise generally in case reports, though as of yet, nothing has shown consistent benefit. Because of the rarity of thymic epithelial tumors, clinical trial enrollment is difficult but nevertheless essential.

Keywords: thymoma, thymic carcinoma, targeted therapy, epidermal growth factor receptor

\section{Introduction}

Thymic epithelial tumors (TETs) are rare neoplasms with a generally good prognosis. While TETs represent the most common cause of anterior mediastinal masses, the incidence of thymoma in the United States is only 0.13 per 100,000 person-years, according to the National Cancer Institute's Surveillance, Epidemiology, and End Results data. ${ }^{1}$ The incidence rate is roughly the same in males and females, and it is most commonly diagnosed in middle age, with the peak incidence of diagnosis occurring in the seventh decade of life. ${ }^{1}$ The majority of patients present with localized disease and are candidates for surgical resection. Most resected patients are cured. However, long-term follow-up is critical, as recurrences can occur decades later. These recurrences usually cannot be approached with curative intent. Therefore, patients with recurrent disease, in addition to those patients who initially present with advanced disease, are candidates for palliative systemic treatments. TETs are best divided into two distinct categories: thymomas (subdivided by the World Health Organization [WHO] classification system) and thymic carcinomas. Thymomas do not appear malignant histologically, but they nevertheless have the capability to recur and metastasize. Fortunately, thymomas tend to be quite responsive to chemotherapy. In contrast, thymic carcinomas have a malignant histologic appearance and a much more aggressive natural history. Relative to thymomas, thymic carcinomas are less chemoresponsive and are more likely to present at a more advanced, unresectable stage.

Alterations affecting chromosome 6p21.3 (MHC locus) and 6q25.2-25.3 are the most frequently observed genetic alterations in thymomas and thymic squamous cell carcinomas. ${ }^{2}$ Additional genetic alterations more common in aggressive subtypes of thymoma are located on chromosomes 5q21-22 (APC locus), 7p15.3, and 8p11.21. Thymic squamous cell carcinomas often show gains of chromosomes 1q, 17q, and 
18 , as well as loss of chromosomes 3p, 6, 16q, and $17 \mathrm{p}$. While epidermal growth factor receptor (EGFR) overexpression is common in thymomas (70\%) and about half (53\%) of thymic carcinomas, EGFR mutations are exceedingly rare, with only three $E G F R$ mutations noted in a total of 158 tumors analyzed. ${ }^{3} R A S$ mutations are also rare, with a series at Memorial Sloan-Kettering Cancer Center showing only three (two thymomas and one thymic carcinoma) out of 45 (7\%) TETs displaying RAS mutations. ${ }^{4}$ KIT expression is frequently observed in thymic carcinomas (79\%) but rarely in thymomas (2\%); however, KIT mutations are seen in only $7 \%$ of thymic carcinomas. ${ }^{3}$

The autoimmune regulator (AIRE) is a gene expressed in a subset of normal thymic epithelial cells. ${ }^{5}$ AIRE promotes the expression of tissue-restricted antigens by medullary thymic epithelial cells, allowing for these cells to delete maturing $\mathrm{T}$ cells with potential for autoreactivity. ${ }^{6}$ AIRE expression is absent in $\sim 95 \%$ of thymomas (the one exception being WHO subtype B1, in which AIRE expression is absent in $40 \%$ of cases). ${ }^{7}$ AIRE deficiency may contribute to the development of autoimmune syndromes such as myasthenia gravis that are commonly seen in patients with thymomas. However, AIRE deficiency alone is not sufficient to cause myasthenia gravis. ${ }^{6}$

Standard chemotherapeutic regimens for TETs consist of platinum and anthracycline, and these combination approaches have response rates (within primarily thymoma cohorts) of between 55\% and 90\%. ${ }^{8-10}$ While initial responses to first-line chemotherapy can be quite durable, ${ }^{11}$ chemotherapy alone holds no curative potential, with the disease destined to recur and progress. While there is no standard salvage option for patients following the failure of platinumbased combination chemotherapy, a multitude of new agents have shown promise in this setting. However, because of the rarity of this neoplasm, phase III studies evaluating treatment efficacy are not available.

\section{Cytotoxic chemotherapy}

Because of the inherent inconveniences of anthracycline-based chemotherapy, including the potential for cardiac toxicity and the inability to administer treatment concurrently with thoracic radiation, interest exists in the evaluation of more convenient, next-generation first-line regimens. As initial TETs were known to demonstrate sensitivity to both platinum ${ }^{12}$ and paclitaxel, ${ }^{13}$ the Eastern Cooperative Oncology Group evaluated the combination of carboplatin and paclitaxel in the hopes that this regimen would demonstrate better outcomes than the anthracycline-based approaches. Unfortunately, the results were disappointing. Forty-six patients with essentially chemotherapy-naïve TETs (one patient had prior remote preoperative chemotherapy) were enrolled in the study and scheduled to receive carboplatin (area under the timeconcentration curve of 6) and paclitaxel $225 \mathrm{mg} / \mathrm{m}^{2}$ every 3 weeks. One patient withdrew consent and never received chemotherapy. Twenty-three patients had thymic carcinoma (including ten patients with WHO subtype B3 disease and 13 patients with WHO subtype $\mathrm{C}$ disease, as defined by the WHO classification system in place at the time of the study). The patients were scheduled to receive a total of six cycles of therapy in the absence of disease progression or excessive toxicity. Forty-nine percent of the 43 evaluable patients completed at least six cycles of chemotherapy without interruption. The treatment was well tolerated: grade 4 neutropenia occurred in $24.4 \%$ of patients and grade 3 sensory neuropathy occurred in $13.3 \%$. Among the patients with thymoma, three achieved a complete response (CR) and six achieved a partial response (PR), according to Response Evaluation Criteria in Solid Tumors guidelines, with an overall response rate (ORR) of $42.9 \%$ (90\% confidence interval [CI], 24.5\%-62.8\%). Ten patients had stable disease. The progression-free survival (median PFS) for the thymoma patients was 16.7 months (95\% CI, 7.2-19.8 months), and the median overall survival (OS) had not been reached after 59.4 months of follow-up. The median duration of response was 16.9 months $(95 \% \mathrm{CI}$, 3.1-22 months). A true response rate of at least $60 \%$ in the thymoma patients was considered the minimum to justify further study. As the upper limit of the CI for response rate barely included $60 \%$, the authors concluded there was limited evidence to support additional investigation of carboplatin and paclitaxel in advanced thymoma.

Among the thymic carcinoma patients, there were no CRs to carboplatin and paclitaxel. Five patients achieved a PR and twelve had stable disease (ORR, 21.7\%; 90\% CI, 9.0\%-40.4\%). The median PFS was 5 months (95\% CI, 3.0-8.3 months) and the median OS was 20.0 months (95\% CI, 5.0-43.6 months). The median duration of response was 4.5 months (95\% CI, 3.4-9.9 months). A true response rate of $45 \%$ was felt to be the minimum to justify further study in thymic carcinoma. Because the response rate within this group was only $21.7 \%$, the authors also concluded that carboplatin and paclitaxel was not a preferred regimen for thymic carcinoma.

In a small Japanese retrospective evaluation of carboplatin and paclitaxel specifically in patients with thymic carcinoma,${ }^{14}$ the results were a little better. Sixteen patients were treated with carboplatin and paclitaxel. Two patients 
demonstrated a CR, four demonstrated a PR, eight had stable disease, and two had progressive disease. The ORR in this study was $37.5 \%$. All patients had stage IV disease or postoperative recurrence of disease. No patients were considered suitable for surgery and/or radiation therapy at the time of chemotherapy initiation. However, two patients received radiation therapy after chemotherapy (and before progression) and one patient underwent surgical resection. Two patients remained disease free for at least 5 years. The median PFS was 8.6 months, while the median OS was 49.4 months.

Carboplatin and paclitaxel is an active regimen for TETs, but it does not appear to be any more active, and may even be less active, than anthracycline-based regimens. Nevertheless, it is a reasonable regimen for patients who are not anthracycline candidates.

Pemetrexed also has activity in TETs. Pemetrexed is a multitargeted antifolate initially approved for the treatment of mesothelioma but now also approved for use in first-line, second-line, and maintenance therapy of nonsquamous non-small cell lung cancer. In a phase II study in patients with previously treated thymoma and thymic carcinoma thus far presented only in abstract form, ${ }^{15,16} \mathrm{a}$ total of 27 patients (16 with thymoma and eleven with thymic carcinoma) received pemetrexed $500 \mathrm{mg} / \mathrm{m}^{2}$ every 3 weeks. The median number of prior treatment regimens received by these patients was two. Pemetrexed treatment was well tolerated, with no grade 4 toxicities. The ORR was $18.5 \%$, and, among the patients with thymoma, two patients achieved a CR and two achieved a PR. One PR rate was observed in a thymic carcinoma patient. The median OS was 29 months for all patients. The median PFS was 45.4 weeks in thymoma patients and 5.1 weeks in thymic carcinoma patients.

In a recent preliminary publication, ${ }^{17}$ the combination of gemcitabine $\left(1000 \mathrm{mg} / \mathrm{m}^{2}\right.$ on days 1 and 8$)\left(1000 \mathrm{~m}^{2}\right)$ and capecitabine $\left(650 \mathrm{mg} / \mathrm{m}^{2}\right.$ twice daily on days $\left.1-14\right)$ was administered to 15 patients with previously treated TETs. Patients involved in the study had received at least two prior chemotherapy regimens. Three patients had thymic carcinoma and the remainder had thymoma. The mean number of cycles administered was seven (median, 6; range, 3-9). Following six cycles, there were three patients who experienced a $\mathrm{CR}$ and three patients who achieved a PR, with an ORR of $40 \%$. One of the PRs occurred in a patient with thymic carcinoma. With a median follow-up period of 22 months, the median PFS was 11 months (95\% CI, 6-17 months) for the patients with thymoma and 6 months
(95\% CI, 3-11 months) for the patients with thymic carcinoma. The median OS had not been reached as of the publication date, but the estimated 1- and 2-year survival rates were $80 \%$ and $67 \%$, respectively. The treatment was well tolerated, with no toxic deaths and no grade 4 toxicities. The most common nonhematologic grade 1-2 toxicities included nausea/vomiting, diarrhea, alopecia, and hand-foot syndrome. One patient experienced grade 3 diarrhea. Twenty percent of patients experienced grade 3 neutropenia and $13 \%$ experienced anemia. Following progression of disease, eleven patients received only supportive care, while two patients received single-agent taxane therapy.

In a recent case report, amrubicin has been shown to lead to a reduction in measurable disease of $12 \%$ in a patient with thymic carcinoma previously treated with two lines of chemotherapy. ${ }^{18}$ In a series of six patients with recurrent thymic carcinoma following platinum-based chemotherapy, two out of six patients treated with salvage platinum and amrubicin achieved a PR. ${ }^{19}$ Single-agent amrubicin in patients with refractory or relapsed thymic malignancies is currently being evaluated (ClinicalTrials.gov Identifier: NCT01364727).

\section{Targeted agents Octreotide}

Somatostatin receptors are expressed in a variety of malignancies including TETs ${ }^{20}$ Octreotide is a potent somatostatin analog with a demonstrated in vitro ability to inhibit thymic epithelial cells. ${ }^{21}$ Long-acting release (LAR) octreotide has demonstrated improved time to tumor progression in metastatic neuroendocrine midgut tumors. ${ }^{22}$ It has been known since the early 1990s that TETs can show evidence of uptake on indium-111-diethylenetriamine pentaacetic acid-D-Phe-labeled octreotide scintigraphy. ${ }^{23}$ The Eastern Cooperative Oncology Group evaluated the efficacy of octreotide in patients with advanced TETs that had activity on octreotide scanning and which were not amenable to curative therapy. ${ }^{24}$ Eligible patients were treated with octreotide at a dose of $0.5 \mathrm{mg}$ subcutaneously three times a day for up to 1 year. Patients with stable disease at the end of 2 months had prednisone $0.6 \mathrm{mg} / \mathrm{kg} /$ day added to their treatment, while those with disease response continued on the octreotide alone and those with progressive disease were removed from the study. Thirty-eight patients were enrolled and eligible, six of whom had thymic carcinoma or carcinoid. Two patients (5.3\%) achieved a CR and ten achieved a PR (25\%), with an ORR of $30.3 \%$. An additional 14 patients had stable disease (36.8\%) and twelve patients (31.6\%) had progressive disease. Four of the PRs occurred within the patients initially treated 
with octreotide alone, while the remaining eight responses (including the two CRs) occurred in the 21 patients in whom prednisone was subsequently added. It should be noted that TETs do respond to steroids alone, but it is unclear if this represents simply a diminishment in the associated lymphoid component of the TET rather than a true response in the neoplastic epithelioid element. ${ }^{25}$ None of the six patients with thymic carcinoma or carcinoid responded to the octreotide alone or in combination with prednisone. The median PFS for patients with thymoma and thymic carcinoma was 8.8 (95\% CI, 3.7-12.3 months) and 4.5 months (95\% CI, 1.9-9.5 months), respectively.

While the Eastern Cooperative Oncology Group study utilized the short-acting formulation of octreotide, the more convenient, long-acting formulation was evaluated in patients with octreotide scan-positive tumors (14 out of 20 patients evaluated). ${ }^{26}$ Patients were dosed with octreotide $20 \mathrm{mg}$ every 2 weeks. Four patients $(28.5 \%)$ showed disease response, five patients $(35.7 \%)$ had stable disease, and two patients $(14.2 \%)$ had progressive disease.

\section{C-KIT inhibitors}

The potential use of imatinib for thymic carcinoma generated a great deal of excitement following the publication of a case report of imatinib leading to an impressive response in a patient with metastatic epidermoid carcinoma of the thymus. ${ }^{27}$ Imatinib is a tyrosine kinase inhibitor (TKI) targeting predominantly Abelson cytoplasmic tyrosine kinase, $(a b l)$ platelet-derived growth factor receptor (PDGFR), and c-KIT. C-KIT (CD117) overexpression is quite common in thymic carcinomas $(\sim 80 \%)$, but it is rare in thymomas $(\sim 4 \%) .{ }^{28}$ The tumor of the patient in the aforementioned imatinib case report did indeed demonstrate overexpression of KIT. In addition, sequencing of the KIT gene also revealed an activating in-frame deletion in exon 11 , the same mutation described for imatinib-responsive gastrointestinal stromal tumors. ${ }^{29}$ Unfortunately, application of imatinib to a broader population of thymic carcinoma patients has not proved as promising. Seven patients with WHO subtype B3/C thymoma were treated with imatinib $600 \mathrm{mg}$ daily, with the dose increasing to $400 \mathrm{mg}$ twice daily if there was no response but also no deterioration after 2 months. ${ }^{30}$ None of the patients had a response, although two did achieve stable disease. It was discovered that three tumors analyzed had no c-KIT or PDGFR mutations, and only one of four tumors analyzed demonstrated c-KIT expression by immunohistochemistry. In another study, eleven patients with previously treated thymic carcinoma, all of whom had tumors that stained for either c-KIT or PDGFR by immunohistochemistry, were treated with imatinib $600 \mathrm{mg}$ daily. ${ }^{31}$ None of the patients responded, although three did have stable disease. In yet another study, 15 patients with TETs, three of whom had thymic carcinomas, were treated with imatinib $400 \mathrm{mg}$ daily. ${ }^{32}$ Two of the thymic carcinoma patients had c-KIT expression on immunohistochemistry. No patient harbored a known c-KIT-activating mutation. While the imatinib was very well tolerated, no radiographic responses were observed. Because of the lack of responses, the study was terminated before it reached its target accrual of 42 patients. It turns out that c-KIT mutations, which may well be required in order for patients to respond to imatinib, are actually rather rare in thymic carcinomas. ${ }^{33,34}$ In a recent analysis of 48 cases of thymic carcinoma, ${ }^{35} 60 \%$ of the tumors expressed c-KIT but only six cases (12.5\%) demonstrated c-KIT mutation. All c-KIT mutations occurred in tumors expressing c-KIT by immunohistochemistry and all occurred in poorly differentiated squamous cell carcinomas with a particularly solid growth pattern. There were a total of twelve such cases in the series, meaning c-KIT mutations were seen in half of them. Therefore, the authors recommended screening cases of thymic carcinoma for c-KIT expression by immunohistochemistry. Positive tumors should be evaluated for c-KIT mutations, and imatinib therapy could be considered for those tumors with likely sensitive mutations in exons 9 or 11 .

\section{Multitargeted kinase inhibitors}

Sorafenib is a multitargeted oral TKI that targets RAF; vascular endothelial growth factor (VEGF) receptors 1, 2, and 3; KIT; PDGF; and FMS-like tyrosine kinase 2. The US Food and Drug Administration (FDA) has approved it for the treatment of renal cell carcinoma and hepatocellular carcinoma. There are two case reports of pretreated patients thymic carcinoma with c-KIT mutations (one in exon 11, the other in exon 17) experiencing impressive responses to sorafenib..$^{36,37}$ In a third case report detailing a durable response in a previously treated patient with thymic carcinoma, the patient's tumor stained positive for KIT and VEGF, but information on mutations within this patient's tumor was not provided. ${ }^{38}$

Sunitinib is another multitarget TKI, blocking VEGF receptors 1-3, FMS-like tyrosine kinase 3, c-KIT, PDGF, and colony-stimulating factor 1 . It is currently FDA approved for the treatment of renal cell carcinoma, gastrointestinal stromal tumor, and well-differentiated pancreatic neuroendocrine tumors. In a trial of sunitinib in 
four patients with metastatic thymic carcinoma refractory to conventional treatment, three patients experienced a PR and one patient had stable disease, according to Response Evaluation Criteria in Solid Tumors guidelines, but with an excellent metabolic response by fluorine-18 fluorodeoxyglucose positron emission tomography. Withdrawal of drug in one patient led to rapid tumor progression, which was then controlled by re-administration of sunitinib. ${ }^{39}$ An analysis of the patients' tumors using polymerase chain reaction and direct sequencing showed no mutations in these patients in $c-K I T, K R A S, B R A F$, or EGFR. All tumors strongly expressed c-KIT and CD5.

SU014813 is an oral multitargeted TKI that targets the VEGF receptors, PDGFRs, KIT, and FMS-like tyrosine kinase 3 . In a recent phase I study, four patients with refractory thymoma were enrolled, and two of them achieved PRs that lasted for 15.3 and 9 months. ${ }^{40}$

\section{EGFR inhibitors}

EGFR immunoreactivity appears to be higher in more aggressive thymomas, but it is not consistently seen in thymic carcinomas. ${ }^{41}$ There are case reports of impressive responses of thymic tumors overexpressing EGFR by immunohistochemistry to cetuximab, a monoclonal antibody against EGFR, ${ }^{42,43}$ and in a series of five patients with refractory thymoma treated with cetuximab, two of five patients responded. ${ }^{44}$ There are as yet no prospective evaluations of cetuximab in TETs, but there is a currently enrolling clinical trial of cetuximab in combination with cisplatin, doxorubicin, and cyclophosphamide prior to surgery in patients with locally advanced or recurrent thymoma (ClinicalTrials.gov Identifier: NCT01025089). The primary outcome measure is the frequency of complete pathologic response.

There is also a case report of a response to erlotinib, an EGFR TKI, in a patient with advanced thymoma. ${ }^{45}$ However, in a phase II study of gefitinib, another EGFR TKI, out of 19 patients with thymoma and seven patients with thymic carcinoma, only one patient experienced a PR. ${ }^{46}$ Fourteen patients experienced stable disease, six of them for longer than 4 months. None of five patients analyzed, including the patient with the PR, were found to have EGFR-activating or KRAS mutations by DNA sequencing. EGFR-activating mutations have been found to be a predictor for response among patients with non-small cell lung cancer. ${ }^{47,48}$ In a phase II study of erlotinib, in conjunction with the VEGF inhibitor bevacizumab, eleven patients with thymoma and seven patients with thymic carcinoma were treated. ${ }^{49}$ No patients achieved a response; stable disease was noted in eleven patients $(60 \%)$. In an evaluation of 20 different subtypes of thymomas and thymic carcinomas, while EGFR positivity by immunohistochemistry was found in the majority of cases $(17 / 20,85 \%)$, none of them were found to have activating mutations in the tyrosine kinase domain comparable with those seen in non-small cell lung cancer. $^{50}$

\section{Histone deacetylase inhibitors}

Cancer cells demonstrate aberrant histone modification. ${ }^{51}$ Histone deacetylase (HDAC) enzymes remove acetyl groups from the terminal lysine residues of histone proteins, which compacts the chromatin and represses associated genes. Therefore, inhibitors of HDAC enzymes alter the patterns of gene expression and can lead to cellular differentiation, growth arrest, and/or apoptosis of cancer cells. ${ }^{52}$ Vorinostat and depsipeptide are HDAC inhibitors approved by the FDA for the treatment of cutaneous T-cell lymphoma.

Belinostat is a pan-HDAC inhibitor. In a phase I study, a patient with thymoma had a minor response lasting 17 months. ${ }^{51}$ In one of the largest phase II trials of a targeted agent in thymic malignancies, belinostat was administered intravenously at a dose of $1 \mathrm{~g} / \mathrm{m}^{2}$ on days $1-5$ of a 21-day cycle until disease progression or intolerance. ${ }^{53}$ Of the 41 patients enrolled, 25 had thymoma and 16 had thymic carcinoma. All patients had progressed following at least one line of platinum-containing chemotherapy. Forty patients were evaluable for response. Two patients, both of whom had thymoma, achieved a PR, for a response rate of $8 \%$ in the thymoma patients (95\% CI, 2.2\%-25\%). Twenty-five patients had stable disease and 13 patients experienced progressive disease. There were no responses among the patients with thymic carcinoma. The median time to progression was 5.8 months, with a median OS of 19.1 months. However, the median survival of the thymic carcinoma patients was significantly worse than for patients with thymoma (median survival rate of 12.4 months versus not yet reached; $P=0.001)$. In the patients with thymoma, the median time to progression was 11.4 months, OS was not reached at 29.3 months. At 6 months, $61 \%$ of patients had not progressed, and $46 \%$ were progression free at 12 months. Eleven patients received twelve or more cycles. While the response rates were not as high as the statisticians had hoped when designing the study (a response rate between $10 \%$ and $30 \%$ was considered worthy of further investigation for this trial), the disease stability was impressive. Toxicity was relatively mild and included hypotension in four patients, nausea (grade 2 ) in three, and 
corrected QT interval prolongation in 13 patients. This was not considered clinical significant but did lead to dose reduction in three patients. Twenty-seven percent of patients received twelve or more cycles. A follow-up phase I/II study of belinostat in combination with cisplatin, doxorubicin, and cyclophosphamide as first-line therapy in patients with advanced thymic malignancies is currently ongoing (ClinicalTrials.gov Identifier: NCT 01100944). Preliminary results in 13 patients, seven with thymoma and six with thymic carcinoma, showed an ORR of $54 \%$, with a $33 \%$ response rate in the thymic carcinoma subgroup. ${ }^{54}$

\section{Insulin-like growth factor-I inhibitors}

An analysis of 132 patients with TETs found that, among 111 samples evaluable for the insulin-like growth factor-1 receptor (IGF-1R) by immunohistochemistry, 20\% were positive. ${ }^{40,55}$ Positivity for IGF-1R was observed more frequently in recurrent disease, in more aggressive histologic subtypes, and in cases of advanced disease. In a phase I study of the anti-IGF-1R monoclonal antibody figitumumab (CP-751871), a patient with metastatic thymoma showed a $10 \%$ reduction in tumor size and stable disease lasting over a year. ${ }^{56}$ In a phase II study of the IGF-1R inhibitor cixutumumab in unresectable TETs that were progressive following at least one platinum-based chemotherapy regimen, ${ }^{57} 49$ patients were enrolled - 37 with thymoma and twelve with thymic carcinoma. Five patients (14\%) of those with thymoma experienced a response, while $28(76 \%)$ had stable disease, and the median PFS was 40 weeks. Within the thymic carcinoma subgroup, there were no responses, five patients (42\%) achieved stable disease, and the median PFS was 6 weeks. Adverse events included hyperglycemia, hyperuricemia, weight loss, lipase elevation, chest wall pain, and aspartate transaminase elevation.

\section{PHA-848I25AC}

PHA-848125AC is an orally administered inhibitor of cyclindependent kinase 2/cyclin A complex and TrkA. TrkA activation promotes tumor growth, and in an analysis of 99 patients with TETs, all but one thymic carcinoma tumor (out of eleven thymic carcinoma tumors) demonstrated cytoplasmic TrkA immunostaining. ${ }^{58}$ In an ongoing phase I trial of this agent, two out of three patients with thymic malignancies demonstrated PRs lasting 6 (patient with thymic carcinoma) and 10 months (patient with WHO subtype B3 thymoma). ${ }^{59}$ Two phase II studies evaluating this agent in thymic carcinoma are ongoing (ClinicalTrials.gov Identifier: NCT01011439, NCT01301391).

\section{Saracatinib}

Steroid receptor coactivator ( $\mathrm{Src}$ ) is a proto-oncogene encoding a non-receptor tyrosine kinase. It is part of a family of enzymes (Src family kinases) involved in cell proliferation and growth, differentiation, angiogenesis, and survival. ${ }^{60}$ Saracatinib (AZD0530) is a Src inhibitor. In a study of this agent in 21 patients with previously treated TETs (14 with thymoma, seven with thymic carcinoma), no responses were observed among the first 13 evaluable patients, so accrual was discontinued. ${ }^{61}$ Eight patients achieved stable disease beyond the first assessment at 56 days. The median PFS was 3.4 months for the thymoma patients and 1.4 months for the thymic carcinoma patients.

\section{Conclusion}

Advanced thymic malignancies are generally responsive to traditional cytotoxic chemotherapy, and reasonable salvage chemotherapy options do exist, although no one regimen can be considered standard. Octreotide has some modest activity and is currently a reasonable treatment option for patients with octreotide-positive scans for disease. To date, other targeted therapies have not shown reliable efficacy when used as single agents, and a consistently present molecular target has not been elucidated. Trials of HDAC and EGFR inhibitors in conjunction with chemotherapy are ongoing, as are single-agent trials of TrkA and IGF-1R inhibitors. Because of the rarity of TETs, clinical trial enrollment is difficult but nevertheless essential.

\section{Disclosure}

The author reports no conflicts of interest in this work.

\section{References}

1. Engels EA. Epidemiology of thymoma and associated malignancies. J Thorac Oncol. 2010;5(10 Suppl 4):S260-S265.

2. Ströbel P, Hohenberger P, Marx A. Thymoma and thymic carcinoma: molecular pathology and targeted therapy. J Thorac Oncol. 2010; 5(10 Suppl 4):S286-S290.

3. Girard N. Thymic tumors: relevant molecular data in the clinic. JThorac Oncol. 2010;5(10 Suppl 4):S291-S295.

4. Girard N, Shen R, Guo T, et al. Comprehensive genomic analysis reveals clinically relevant molecular distinctions between thymic carcinomas and thymomas. Clin Cancer Res. 2009;15(22):6790-6799.

5. Heino M, Peterson P, Kudoh J, et al. Autoimmune regulator is expressed in the cells regulating immune tolerance in thymus medulla. Biochem Biophys Res Commun. 1999;257(3):821-825.

6. Marx A, Hohenberger P, Hoffmann H, et al. The autoimmune regulator AIRE in thymoma biology: autoimmunity and beyond. JThorac Oncol. 2010;5(10 Suppl 4):S266-S272.

7. Ströbel P, Murumägi A, Klein R, et al. Deficiency of the autoimmune regulator AIRE in thymomas is insufficient to elicit autoimmune polyendocrinopathy syndrome type 1 (APS-1). J Pathol. 2007;211(5):563-571.

8. Fornasiero A, Daniele O, Ghiotto C, et al. Chemotherapy for invasive thymoma: a 13-year experience. Cancer. 1991;68(1):30-33. 
9. Loehrer PJ Sr, Kim K, Aisner SC, et al; for Eastern Cooperative Oncology Group; Southwest Oncology Group; Southeastern Cancer Study Group. Cisplatin plus doxorubicin plus cyclophosphamide in metastatic or recurrent thymoma: final results of an intergroup trial. J Clin Oncol. 1994;12(6):1164-1168.

10. Loehrer PJ Sr, Chen M, Kim K, et al. Cisplatin, doxorubicin, and cyclophosphamide plus thoracic radiation therapy for limited-stage unresectable thymoma: an intergroup trial. J Clin Oncol. 1997;15(9):3093-3099.

11. Loehrer PJ, Wang W, Aisner S, et al. Long-term follow-up of patients with locally advanced or metastatic thymic malignancies: the Eastern Cooperative Oncology Group (ECOG) experience. Paper presented at: 40th American Society of Clinical Oncology Annual Meeting; June 5-8, 2004; New Orleans, LA

12. Bonomi PD, Finkelstein D, Aisner S, Ettinger D. EST 2582 phase II trial of cisplatin in metastatic or recurrent thymoma. Am J Clin Oncol. $1993 ; 16(4): 342-345$

13. Umemura S, Segawa Y, Fujiwara K, et al. A case of recurrent metastatic thymoma showing a marked response to paclitaxel monotherapy. Jpn J Clin Oncol. 2002;32(7):262-265.

14. Furugen M, Sekine I, Tsuta K, et al. Combination chemotherapy with carboplatin and paclitaxel for advanced thymic cancer. Jpn J Clin Oncol. 2011;41(8):1013-1016.

15. Loehrer PJ Sr, Yiannoutsos CT, Dropcho S, et al. A phase II trial of pemetrexed in patients with recurrent thymoma or thymic carcinoma. J Clin Oncol. 2006;24(18S):Abstract 7079.

16. Schmitt J, Loehrer PJ Sr. The role of chemotherapy in advanced thymoma. J Thorac Oncol. 2010;5(10 Suppl 4):S357-S360.

17. Palmieri G, Merola G, Federico P, et al. Preliminary results of phase II study of capecitabine and gemcitabine (CAP-GEM) in patients with metastatic pretreated thymic epithelial tumors (TETs). Ann Oncol. 2010;21(6):1168-1172.

18. Igawa S, Yanagisawa N, Makita S, et al. A case of recurrent invasive thymic cancer showing a stable disease to carboplatin plus paclitaxel Gan To Kagaku Ryoho. 2010;37(11):2161-2163. Japanese.

19. Koizumi T, Takabayashi Y, Yamagishi S, et al. Chemotherapy for advanced thymic carcinoma: clinical response to cisplatin, doxorubicin, vincristine, and cyclophosphamide (ADOC chemotherapy). Am J Clin Oncol. 2002;25(3):266-268.

20. Krenning EP, Kwekkeboom DJ, Bakker WH, et al. Somatostatin receptor scintigraphy with [111In-DTPA-D-Phe1]- and [123I-Tyr3]octreotide: the Rotterdam experience with more than 1000 patients. Eur J Nucl Med. 1993;20(8):716-731.

21. Ferone D, van Hagen PM, van Koetsveld PM, et al. In vitro characterization of somatostatin receptors in the human thymus and effects of somatostatin and octreotide on cultured thymic epithelial cells. Endocrinology. 1999;140(1):373-380.

22. Rinke A, Müller HH, Schade-Brittinger C, et al. Placebo-controlled, double-blind, prospective, randomized study on the effect of octreotide LAR in the control of tumor growth in patients with metastatic neuroendocrine midgut tumors: a report from the PROMID Study Group. J Clin Oncol. 2009;27(28):4656-4663.

23. Lastoria S, Vergara E, Palmieri G, et al. In vivo detection of malignant thymic masses by indium-111-DTPA-D-Phe1-octreotide scintigraphy. J Nucl Med. 1998;39(4):634-639.

24. Loehrer PJ Sr, Wang W, Johnson DH, Aisner SC, Ettinger DS. Octreotide alone or with prednisone in patients with advanced thymoma and thymic carcinoma: an Eastern Cooperative Oncology Group Phase II Trial. J Clin Oncol. 2004;22(2):293-299.

25. Basha AM, Bonomi PD. Glucocorticoid therapy for invasive thymoma progressing after chemotherapy: a review of 4 cases. Paper presented at: 38th American Society of Clinical Oncology Annual Meeting; May 18-21, 2002; Orlando, FL.

26. Zampa G. Targeting the therapy: octreotide in thymoma relapse. J Clin Oncol. 2007;25(18S):Abstract 18199.

27. Ströbel P, Hartmann M, Jakob A, et al. Thymic carcinoma with overexpression of mutated KIT and the response to imatinib. NEngl J Med. 2004;350(25):2625-2626.
28. Nakagawa K, Matsuno Y, Kunitoh H, Maeshima A, Asamura H, Tsuchiya R. Immunohistochemical KIT (CD117) expression in thymic epithelial tumors. Chest. 2005;128(1):140-144.

29. Allander SV, Nupponen NN, Ringnér M, et al. Gastrointestinal stromal tumors with KIT mutations exhibit a remarkably homogeneous gene expression profile. Cancer Res. 2001;61(24):8624-8628.

30. Giaccone G, RajanA, Ruijter R, SmitE, van Groeningen C, Hogendoorn PC. Imatinib mesylate in patients with WHO B3 thymomas and thymic carcinomas. J Thorac Oncol. 2009;4(10):1270-1273.

31. Salter JT, Lewis D, Yiannoutsos CT, Loehrer PJ, Risley L, Chiorean EG. Imatinib for the treatment of thymic carcinoma. J Clin Oncol. 2008;26S:Abstract 8116.

32. Palmieri G, Marino $\mathrm{M}$, Buonerba $\mathrm{C}$, et al. Imatinib mesylate in thymic epithelial malignancies. Cancer Chemother Pharmacol. 2012;69(2):309-315.

33. Tsuchida M, Umezu H, Hashimoto T, et al. Absence of gene mutations in KIT-positive thymic epithelial tumors. Lung Cancer. 2008;62(3): 321-325.

34. Yoh K, Nishiwaki Y, Ishii G, et al. Mutational status of EGFR and KIT in thymoma and thymic carcinoma. Lung Cancer. 2008;62(3):316-320.

35. Schirosi L, Nannini N, Nicoli D, et al. Activating c-KIT mutations in a subset of thymic carcinoma and response to different c-KIT inhibitors. Ann Oncol. Epub February 21, 2012.

36. Bisagni G, Rossi G, Cavazza A, Sartori G, Gardini G, Boni C. Long lasting response to the multikinase inhibitor bay 43-9006 (sorafenib) in a heavily pretreated metastatic thymic carcinoma. J Thorac Oncol. 2009;4(6):773-775.

37. Dişel U, Oztuzcu S, Beşen AA, et al. Promising efficacy of sorafenib in a relapsed thymic carcinoma with c-KIT exon 11 deletion mutation. Lung Cancer. 2011;71(1):109-112.

38. Li XF, Chen Q, Huang WX, Ye YB. Response to sorafenib in cisplatin-resistant thymic carcinoma: a case report. Med Oncol. 2009;26(2):157-160.

39. Ströbel P, Bargou R, Wolff A, et al. Sunitinib in metastatic thymic carcinomas: laboratory findings and initial clinical experience. $\mathrm{Br} J$ Cancer. 2010;103(2):196-200.

40. Fiedler W, Giaccone G, Lasch P, et al. Phase I trial of SU14813 in patients with advanced solid malignancies. Ann Oncol. 2011;22(1):195-201.

41. Aisner SC, Dahlberg S, Hameed MR, et al. Epidermal growth factor receptor, c-KIT, and HER2/neu immunostaining in advanced or recurrent thymic epithelial neoplasms staged according to the 2004 World Health Organization in patients treated with octreotide and prednisone: an Eastern Cooperative Oncology Group study. J Thorac Oncol. 2010;5(6):885-892.

42. Farina G, Garassino MC, Gambacorta M, La Verde N, Gherardi G, Scanni A. Response of thymoma to cetuximab. Lancet Oncol. 2007;8(5): 449-450.

43. Palmieri G, Marino M, Salvatore M, et al. Cetuximab is an active treatment of metastatic and chemorefractory thymoma. Front Biosci. 2007; 12:757-761.

44. Palmieri G, Montella L, Merola G, et al. Clinical outcome of patients with thymic epithelial tumors. J Clin Oncol. 2007;25(18S):Abstract 14148.

45. Christodoulou C, Murray S, Dahabreh J, et al. Response of malignant thymoma to erlotinib. Ann Oncol. 2008;19(7):1361-1362.

46. Kurup A, Burns M, Dropcho S, Pao W, Loehrer PJ. Phase II study of gefitinib treatment in advanced thymic malignancies. J Clin Oncol. 2005;23(16S):Abstract 7068.

47. Lynch TJ, Bell DW, Sordella R, et al. Activating mutations in the epidermal growth factor receptor underlying responsiveness of non-small-cell lung cancer to gefitinib. $N$ Engl J Med. 2004;350(21):2129-2139.

48. Mok TS, Wu YL, Thongprasert S, et al. Gefitinib or carboplatinpaclitaxel in pulmonary adenocarcinoma. NEngl J Med. 2009;361(10): 947-957.

49. Bedano PM, Perkins S, Burns M, et al. A phase II trial of erlotinib plus bevacizumab in patients with recurrent thymoma or thymic carcinoma. J Clin Oncol. 2008;26S:Abstract 19087. 
50. Meister M, Schirmacher P, Dienemann H, et al. Mutational status of the epidermal growth factor receptor (EGFR) gene in thymomas and thymic carcinomas. Cancer Lett. 2007;248(2):186-191.

51. Steele NL, Plumb JA, Vidal L, et al. A phase 1 pharmacokinetic and pharmacodynamic study of the histone deacetylase inhibitor belinostat in patients with advanced solid tumors. Clin Cancer Res. 2008;14(3):804-810.

52. Marks PA, Richon VM, Rifkind RA. Histone deacetylase inhibitors: inducers of differentiation or apoptosis of transformed cells. $J$ Natl Cancer Inst. 2000;92(15):1210-1216.

53. Giaccone G, Rajan A, Berman A, et al. Phase II study of belinostat in patients with recurrent or refractory advanced thymic epithelial tumors. J Clin Oncol. 2011;29(15):2052-2059.

54. Thomas A, Rajan A, Khozin S, et al. A phase (Ph) I/II study of belinostat (Bel) in combination with cisplatin, doxorubicin, and cyclophosphamide (PAC) in the first-line treatment of advanced or recurrent thymic malignancies. Paper presented at: 48th American Society of Clinical Oncology Annual Meeting; June 1-5, 2012; Chicago, IL.

55. Zucali PA, Petrini I, Lorenzi E, et al. Insulin-like growth factor-1 receptor and phosphorylated AKT-serine 473 expression in 132 resected thymomas and thymic carcinomas. Cancer. 2010;116(20): 4686-4695.
56. Haluska P, Shaw HM, Batzel GN, et al. Phase I dose escalation study of the anti insulin-like growth factor-I receptor monoclonal antibody CP-751,871 in patients with refractory solid tumors. Clin Cancer Res. 2007;13(19):5834-5840.

57. Rajan A, Riely GJ, Carter CA, et al. Phase II study of cixutumumab (IMC-A12) in thymic malignancies. Paper presented at: 48th American Society of Clinical Oncology Annual Meeting; June 1-5, 2012; Chicago, IL

58. Kim DJ, Yang WI, Kim SH, Park IK, Chung KY. Expression of neurotrophin receptors in surgically resected thymic epithelial tumors. Eur J Cardiothorac Surg. 2005;28(4):611-616.

59. Rajan A, Berman A, Kelly RJ, et al. Phase II study of the insulin-like growth factor-1 receptor (IGF-1R) antibody cixutumumab (C) in patients (pts) with thymoma (T) and thymic carcinoma (TC). J Clin Oncol. 2010;28S:Abstract e17525.

60. Wheeler DL, Iida M, Dunn EF. The role of Src in solid tumors. Oncologist. 2009;14(7):667-678.

61. Wakelee HA, Gubens MA, Burns M, et al. A phase II study of saracatinib (AZD0530), a Src inhibitor, administered orally daily to patients with advanced thymic malignancies. Paper presented at: Chicago Multidisciplinary Symposium in Thoracic Oncology; December 9-11, 2010; Chicago, IL.
OncoTargets and Therapy

\section{Publish your work in this journal}

OncoTargets and Therapy is an international, peer-reviewed, open access journal focusing on the pathological basis of all cancers, potential targets for therapy and treatment protocols employed to improve the management of cancer patients. The journal also focuses on the impact of management programs and new therapeutic agents and protocols on

\section{Dovepress}

patient perspectives such as quality of life, adherence and satisfaction. The manuscript management system is completely online and includes a very quick and fair peer-review system, which is all easy to use. Visit http://www.dovepress.com/testimonials.php to read real quotes from published authors. 